\title{
Estimating Child Death from Perinatal Originating Conditions and Congenital Malformations in Thailand
}

\author{
Binita K. PAUDEL ${ }^{1,2}$, Apiradee LIM ${ }^{2}$, \\ Arjun Mani GURAGAN ${ }^{3,4, *}$ and Jagadish PAUDEL ${ }^{4}$ \\ ${ }^{1}$ Purbanchal University College of Medical and Allied Science (PUCMAS), Biratnagar, Nepal \\ ${ }^{2}$ Department of Mathematics and Computer Science, Faculty of Science and Technology, \\ Prince of Songkla University, Pattani Campus, Pattani 94000, Thailand \\ ${ }^{3}$ Initiative for Research, Education and Community Health (InREACH-Nepal), Kathmandu \\ ${ }^{4}$ Civic Independence Development (CID)-Nepal, Kathmandu, Nepal
}

('Corresponding author's e-mail: arjunmg@gmail.com)

Received: 8 June 2018, Revised: 23 September 2018, Accepted: 20 October 2018

\begin{abstract}
The objective of this study is to examine the correctness of reported causes of deaths in children and estimate deaths from perinatal originating conditions (ICD-10, chapter block P) and congenital malformations (ICD-10, chapter block Q) in Thailand. The subset of Thai deaths of children under 5 years old (149 from a total of 9964 deaths) in a 2005 Verbal Autopsy (VA) study was selected as a sample; the data from 1996 - 2009 were used as the target population for this study. Three different logistic regression models were conducted for 3 outcomes of interest: deaths from Perinatal Originating Conditions (POC), deaths from Congenital Malformations (CM) and from other causes including the effects of province, sex and the location of death. This study found no difference in the probability of dying from the POC and CM by sex and province. As expected, the study found significant differences in reporting the correct causes of death inside and outside the hospital. Nearly $90 \%$ of deaths from both POC and CM that occurred in hospital were reported correctly. In contrast, only 60 and $70 \%$, of these causes of deaths that occurred outside hospital were reported correctly. The number of child deaths from POC in the 14 year period was estimated at 36,838 and from CM was estimated at 26,806. These estimations of deaths from POC are 1.49 times more and from CM are 2.18 times higher than the reported numbers.
\end{abstract}

Keywords: Perinatal originating condition, Congenital malformation, Verbal autopsy, Logistic regression, Thailand

\section{Introduction}

There was a significant decline of under 5 mortality rates in all regions of the globe between 1990 and 2008 [1]. Mortality remains high during the $1^{\text {st }}$ month of life (neonatal period), and relatively little progress has been made in reducing neonatal mortality. However, the distribution of reported causes of deaths in neonates varies substantially between countries. The major direct causes of neonatal deaths at the global level are infections including tetanus (35\%), preterm birth (28\%) and birth asphyxia $(23 \%)$ [2]. Based on the International Statistical Classification of Disease, $10^{\text {th }}$ revision (ICD-10), the above mentioned causes and conditions other than infection are listed in chapter 16 (P00-P96) entitled "Certain conditions originating in the perinatal period" $(\mathrm{P})$ and in chapter 17 (Q00-Q99) entitled "Congenital malformations, deformations and chromosomal abnormalities" $(\mathrm{Q})$. Both conditions $(\mathrm{P}+\mathrm{Q})$ are directly related to pregnancy, labor and delivery. Such conditions are easily preventable and manageable with 
proper prenatal, antenatal care and safe delivery related programs. Child survival efforts can be effective only if they are based on accurate information about causes of deaths [3]. Therefore, authors choose these 2 major causes of child death for further analysis and estimation.

Vital Registration (VR) is the main source of information on cause of death by age and sex. Yet, in Thailand, the death records are considered as incomplete and inaccurate. About $40 \%$ of death certificates in Thailand give the cause of death as "ill-defined" [4] and this severely limits their public utility. In $2004,65 \%$ of registered deaths ( $70 \%$ in 2003) occurred outside the hospital [5]. In particular, child death is believed to be reported in about $50 \%$ of cases [6]. In such a scenario, Verbal Autopsy (VA) is one of the best recommended methods to determine individuals' cause of death and is based on an interview with next of kin or other guardians. In recent years, verbal autopsy studies have been widely used to provide information on the cause of death in areas where civil registration and death certification systems are weak, and where most people die at home without having had contact with the health system [7-10]. Therefore, a verbal autopsy (VA) study was carried out to verify the causes of death in 2005 based on a sample of 3316 in-hospital and 6328 outside-hospital deaths from 28 selected districts in 9 provinces [4]. However, VA data have been analyzed elsewhere and published in some articles [4,6,11,12]. After reviewing all these articles, we found more scope for analyzing the same data and to develop an appropriate method to estimate deaths in the few years before and after the VA study. Using new methods, in the data, some papers have already been published focusing on deaths due to HIV/AIDS, transport accident and cancer in Thailand [13-15] and some others are in the process of review for publication. This paper is a part of the same study focusing only on 2 causes of child deaths as stated above in the $1^{\text {st }}$ paragraph. Hence, the objectives of our study were 1) to examine the correctness of the reported causes of deaths in children aged less than 5 years old and, if necessary, allocate them into a correct cause group based on the 2005 verbal autopsy study. 2) To estimate the number of child deaths caused from perinatal originating conditions and congenital malformations during 1996 - 2009. It is expected that the result of this study will provide some insight into the trend of child mortality and reporting system in Thailand. Evidence based knowledge on the cause of death can affect national policies on intervention and care, and related resource allocation.

\section{Materials and methods}

This study of child deaths in Thailand is based on data from 2 sources. One source is from a large scale field Verbal Autopsy (VA) study conducted in 2005 to verify the registered causes of death in a nationally representative sample of 11,984 , approximately $2.5 \%$ of all registered deaths [6]. The other source of data was the Vital Registration (VR) database for 14 years from 1996 - 2009 obtained from the Bureau of Policy and Strategy database, the Ministry of Public Health. It is noted from Table 1 that all deaths occurred inside and outside hospital due to POC and more than $2 / 3$ of deaths due to CM occurred in the under 5 aged group whereas almost all deaths by other causes were occurred in $5+$ years age group. Therefore, this study developed a model using the subset of under 5 year old cases from the VA study and extended this model to the relevant target population from the VR database as a means of valid estimation.

The VA study provided a data table, including 6 fields which relate to the deceased person's province, gender, age, location of death, ICD-10 code reported on the death certificate and VA-assessed ICD-10 code. Considering the distribution of reported and VA-assessed deaths in 2005, we defined 3 major causes of death groups based on chapter-block classification of ICD-10 codes 1) all the deaths under chapter XVI block P00-P96 "Perinatal Originating Causes", 2) chapter XVII block Q00-Q99 as "Congenital Causes" and 3) the remaining 20 chapters as "Other Causes". Province, gender, location of death and reported ICD-10 code were considered as determinants. 
Table 1 Distribution of under 5 year old deaths in VA study sample by major cause groups in Thailand, 2005.

\begin{tabular}{lccr}
\hline Cause group & $\mathbf{0}-\mathbf{4}$ years & 5+ years & Total \\
\hline Perinatal (ICD-10 block P) & $59(49+10)$ & 0 & 59 \\
Congenital (ICD-10 block Q) & $38(27+11)$ & 16 & 54 \\
Other causes & $52(28+24)$ & 9479 & 9531 \\
Total & $\mathbf{1 4 9}$ & $\mathbf{9 4 9 5}$ & $\mathbf{9 6 4 4}$ \\
\hline
\end{tabular}

Note: The numbers in the parenthesis are the number of deaths occurred inside and outside the hospital respectively

Logistic Regression Model: Three sets of logistic regression models were fitted for the 3 cause groups mentioned above. This model formulates the probability $P$ that a person died from the selected cause as an additive linear function of the 3 determinant factors as follows:

$\ln \left[\frac{P_{i j k}}{1-P_{i j k}}\right]=\mu+a_{i}+\beta_{j}+\gamma_{k}$

where, $p$ is the probability of children deaths, $\mu$ is a constant, and $\alpha, \beta, \gamma$ are parameters namely province, gender group, VR cause location group $i=1-9$ province groups, $j=1-2$ gender groups, $k=1$ - 4 VR cause-location groups.

The province factor has 9 levels, one for each province in the VA sample. For the prediction of death from specific causes, we define a VR cause-location group comprising the VR cause group and location of death (inside or outside hospital). We created only 2 cause groups where one group is the cause of interest and the "other" group aggregated deaths from all other causes including "ill define". Therefore, VR cause-location group has 4 levels.

Finally, we applied the model to the target population assuming that the model is valid before and after 2005. As we aggregated results from three separate logistic regression models, there was a small discrepancy in estimated death counts for each year's total count. Simple scaling was applied to match the estimated counts with the total reported counts.

\section{Results and discussion}

Of the total 9644 VA samples, $149(1.55 \%)$ deaths were aged under 5 years. Of the total under 5 year's deaths, $39.6 \%$ deaths were by the POC and $25.5 \%$ of deaths were due to CM. The bubble graph in Figure 1 shows the cross tabulation between the reported causes of death from VR and the verified causes of death from the VA study. The 3 large bubbles along the diagonal represent the number of correctly reported causes of death that matched with the cause of deaths assessed by the VA study. About $68 \%(40 / 59)$ of deaths from POC and $54 \%$ (21/38) deaths from CM were reported correctly; all other bubbles represent the incorrect reporting of the causes of death. So $28 \%$ of the total causes of death were reported incorrectly including $11 \%$ as ill-defined. Twelve deaths from each POC and CM were underrepresented in the VR reports, so 24 deaths from other causes were over-represented. 


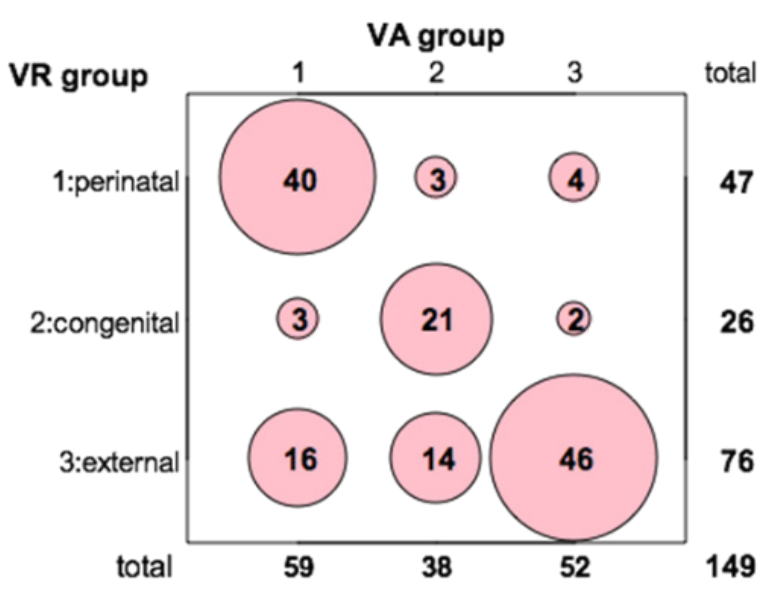

Figure 1 Cross tabulation matrix on cause of death of under 5 children reported in vital registration and assessed in a 2005 Thai verbal autopsy presented in bubble plot. The size of the bubble denotes the population size.

Figure 1 simply describes the observed percentage of reported and verified cause of death whereas Figure 2 shows the crude and adjusted percentages of reported child death from the 3 major causes by province, sex and reported cause of death both from inside and outside the hospital. The adjusted percentages were obtained from logistic model. The average percentage of deaths from perinatal originating conditions and congenital malformation were 39.6 and $25.5 \%$, respectively. About $50 \%$ of deaths in both ChangRai (Id no 6) and Songkla (Id no 9) were from POC while only $12 \%$ deaths in Chumpon (Id no 8) were from POC. However, after comparing the adjusted percentages with overall means using $95 \% \mathrm{CI}$, the probability of dying from POC or CM did not vary significantly by sex or province. Nearly $90 \%$ of deaths from both perinatal originating conditions and congenital malformation that occurred in the hospital were reported correctly. In contrast, only 60 and $70 \%$ of deaths from respective causes that occurred outside the hospital were reported correctly. However, among the cases of deaths, both inside and outside hospital, 10 - $20 \%$ of those reported as other causes came from POC or CM. As expected, there were big significant differences in the report of the correct cause of death between inside and outside the hospital.

The area plot in Figure 3 shows the reported and estimated number of child deaths after adjusting for mis-reporting based on the models. The area of each color strip denotes the number of deaths in each group. Using this method we found that over the 14 years period from 1996 to 2009, the estimated number of child deaths from POC was 36,838 and from CM was 26,806. These estimations are 1.49 and 2.18 times higher than the reported totals of 24,770 and 12,277 , respectively. However, for other causes the estimation was lower by 1.5 times, mainly due to being reported as "ill-defined". The estimated numbers of deaths from the POC and CM were found 1.65 and 2.18 times higher, respectively, than reported number for males while they were 1.32 and 2.26 times higher than the reported number for females. The variation in the number of child deaths between male and female was not significantly different. 

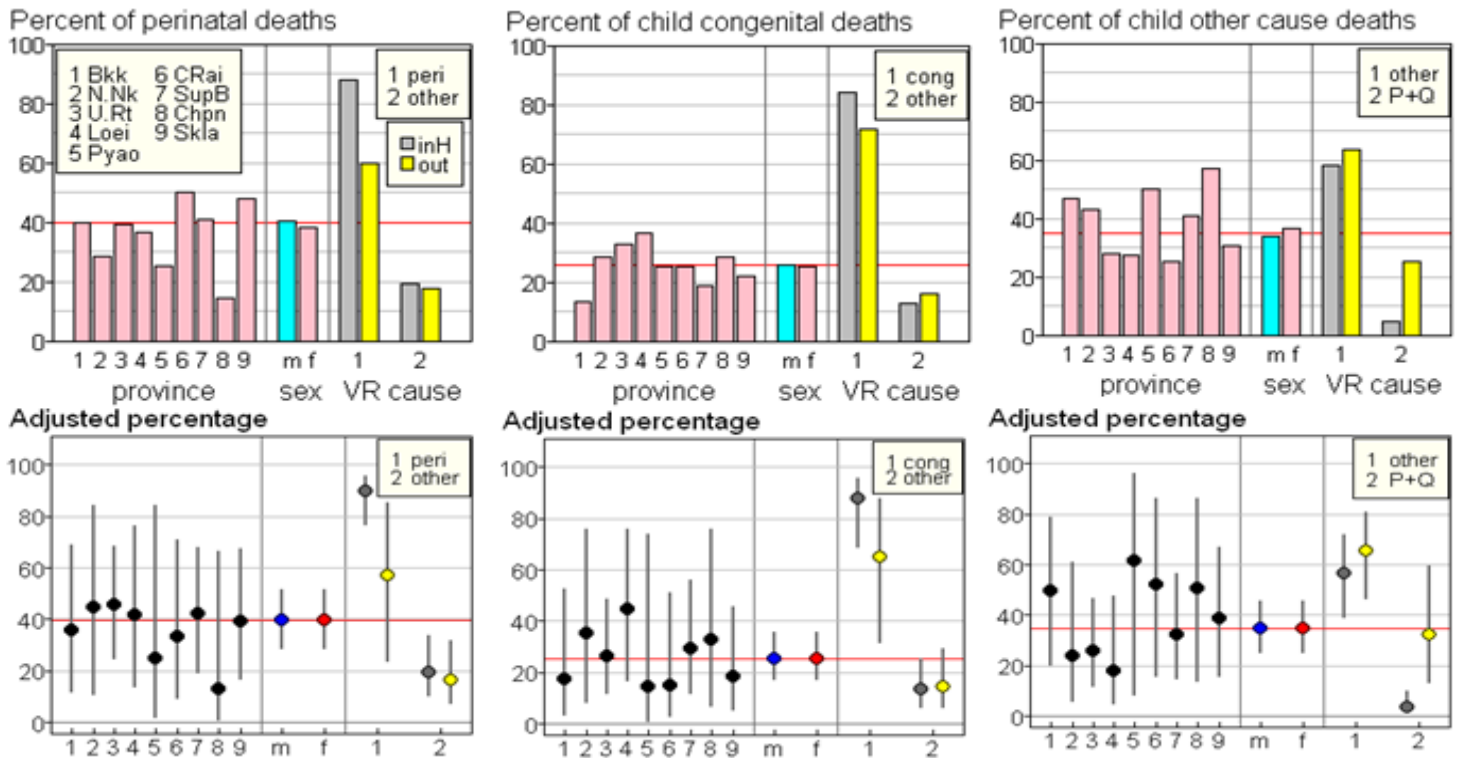

Figure 2 Crude and adjusted percent distribution of reported child death from perinatal originating conditions, congenital malformation and other causes by province, sex and reported causes in and outside the hospital deaths from the 2005 verbal autopsy study.
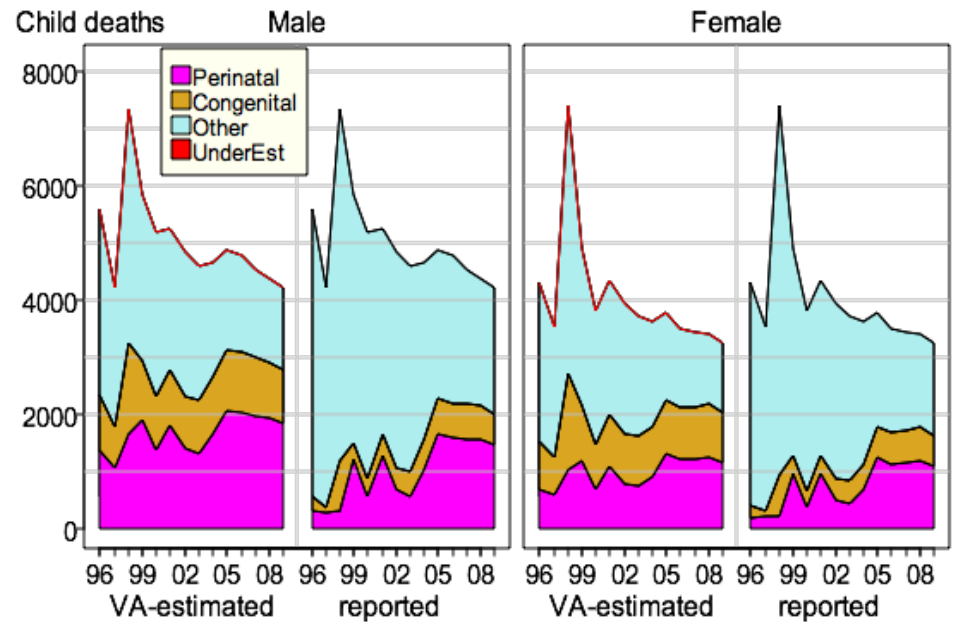

Figure 3 Comparison between reported and estimated number of child deaths from the years 1996 - 2009 after adjusting misreports based on models created from the Thai verbal autopsy study in 2005.

We applied the model developed in this study to estimate the number of deaths over the 14 years period from 1996 to 2009. Table 2 shows the number of reported and estimated deaths from model and the inflation ratio between reported and estimated deaths. The inflation ratio indicates the discrepancy between reported and estimated number of deaths. Although the number of reported deaths increased every year, the inflation ratio in both groups have decreased from more than 4 to much less than 2 over the period between 1996 - 2009 (Table 2). 
Table 2 Ratio between reported deaths from VR and estimated deaths using the VA model are due to perinatal originating condition and congenital malformation amongst under five-year-old children in Thailand.

\begin{tabular}{ccccccc}
\hline \multirow{2}{*}{ Year } & \multicolumn{2}{c}{ Deaths from perinatal originating condition } & \multicolumn{3}{c}{ Deaths from congenital malformation } \\
\cline { 2 - 6 } & Reported & Estimated & Ratio(IF) & Reported & Estimated & Ratio(IF) \\
\hline 1996 & 521 & 1969 & 3.78 & 450 & 1736 & 3.86 \\
1997 & 499 & 1581 & 3.17 & 203 & 1321 & 6.51 \\
1998 & 569 & 2625 & 4.61 & 1576 & 3266 & 2.07 \\
1999 & 2202 & 3028 & 1.38 & 594 & 1983 & 3.34 \\
2000 & 973 & 1999 & 2.05 & 566 & 1688 & 2.98 \\
2001 & 2279 & 2872 & 1.26 & 685 & 1866 & 2.72 \\
2002 & 1198 & 2149 & 1.79 & 735 & 1751 & 2.38 \\
2003 & 1027 & 2028 & 1.97 & 814 & 1739 & 2.14 \\
2004 & 1731 & 2543 & 1.47 & 951 & 1834 & 1.93 \\
2005 & 2946 & 3384 & 1.15 & 1116 & 2002 & 1.79 \\
2006 & 2738 & 3235 & 1.18 & 1155 & 1971 & 1.71 \\
2007 & 2737 & 3201 & 1.17 & 1171 & 1932 & 1.65 \\
2008 & 2781 & 3204 & 1.15 & 1164 & 1905 & 1.64 \\
2009 & 2569 & 3019 & 1.18 & 1097 & 1812 & 1.65 \\
\hline
\end{tabular}

\section{Discussion}

The correct cause of death is key to guide public health programming. Evidence based knowledge on the cause of death affect national policies on intervention and care, and related resource allocation. There are mainly 2 issues related to child death registration in Thailand. The first issue, as mentioned in the introduction section, is only half of the children's deaths have been reported in the vital registration system. Another issue is the correct way of reporting the cause of death in the vital registration system. This study focuses on the $2^{\text {nd }}$ issue and estimates the number of deaths due to perinatal originating condition and congenital malformation after correcting the reported cause of deaths. When we compared the reported cause of deaths with validated causes of death from a VA study, we found that more than one quarter of the reported causes of death in children were incorrectly reported in Thailand, in 2005 . All the deaths that occurred in hospital were also not reported correctly; however, the proportion of deaths correctly reported inside the hospital was significantly higher than the proportion of deaths correctly reported outside the hospital. About 40 and $30 \%$ of deaths from both POC and CM occurred outside the hospital were reported incorrectly in 2005. Most of the deaths from both POC and CM were incorrectly reported as ill-defined, followed by respiratory or circulatory cause. This is obvious, not only in the cause of death amongst children, but also in all causes of deaths because about $40 \%$ of death certificates in Thailand give the cause of death as "ill-defined" [4]. 
This study allocated the incorrect reported causes of death in under 5 year old children to their correct ICD chapter-block groups based on a logistic model developed from the 2005 VA study. After the results of our logistic regression model, applied to the reference population, the deaths from POC and CM were found to be 1.49 times and 2.18 times higher than those reported in VR respectively. Klinjun et al. [14] also found 1.68 to 2.65 times higher estimation in different gender/age groups due to a transport accident in Thailand in 2005. Although the child death rate has decreased substantially in recent years in Thailand, the number of reported child deaths has increased over the 14 years period. This increasing trend in the number of reported deaths and decreasing trend in inflation ratio between estimated and reported number of deaths from POC and CM, from 1996 to 2009, supports that the quality of vital registration system has improved in Thailand over this period (Table 2). This study used the logistic regression model including demographic factor as recommended by Chutinantakul et al. [13], because this method provided a more accurate estimation comparing a simple cross-referencing method in HIV estimation. Such methods were also used to estimate deaths from the transport accident [14] and liver cancer [15] in Thailand. However, this estimation method cannot address the underreporting of about $50 \%$ of deaths amongst children, as it was only based on the reported number of deaths. The focus of the verbal autopsy study was on all causes of death; the sample was nationally representative for overall causes of deaths from all age groups and sex [6]. Therefore, it was the best available VA data to understand more about the number of child deaths due to different causes in Thailand. However, the study has some limitations in terms of sample size and verbal autopsy tools. We were aware of these limitations and considered them while analyzing and interpreting the results. We recommended to conduct a Verbal autopsy study using more specific VA tools to assess the cause of deaths in children.

\section{Acknowledgements}

The authors are grateful to Emeritus Professor Don McNeil, Macquarie University, Australia for his supervision and invaluable guidance. The Authors also would like to thank the Thai Ministry of Public Health for providing the data. The Principle Investigator is also thankful to the Graduate school of Prince of Songkla University for providing the PhD fellowship and thesis support grant.

\section{References}

[1] United Nations Development Group. Thematic paper on MDG 4,5 and 6. Available at: http://www.undg.org/docs/11421/MDG4-6_1954-UNDG-MDG456-LR.pdf, accessed April 2010.

[2] JE Lawn, K Wilczynska-Ketende and SN Cousens. Estimating the causes of 4 million neonatal deaths in the year 2000. Int. J. Epidemiol. 2006; 35, 706-18.

[3] J Bryce, C Boschi-Pinto, K Shibuya, RE Black and WHO Child Health Epidemiology Reference Group. WHO estimates of the causes of death in children. Lancet 2005; 365, 1147-52.

[4] C Rao, Y Porapakkham, J Pattaraarchachai, W Polprasert, N Swampunyalert and AD Lopez. Verifying causes of death in Thailand: Rationale and methods for empirical investigation. Popul. Health Metrics 2010; 8, 11.

[5] V Tangcharoensathien, P Faramnuayphol, W Teokul, K Bundhamcharoen and S Wibulpholprasert. A critical assessment of mortality statistics in Thailand: potential for improvements. Bull. World Health Organ. 2006; 84, 233-8.

[6] Y Porapakkham, C Rao, J Pattaraarchachai, W Polprasert, T Vos, T Adair and AD Lopez. Estimated causes of death in Thailand, 2005: Implications for health policy. Popul. Health Metrics 2010; 8, 14.

[7] M Anker, RE Black, C Coldham, HD Kalter, MA Quigley, D Ross and RW Snow. A Standard Verbal Autopsy Method for Investigating Causes of Death in Infants and Children, Availble at: http://www.who.int/csr/resources/publications/surveillance/whocdscsrisr994.pdf, accessed May 2007. 
[8] AC Lee, LC Mullany, JM Tielsch, J Katz, SK Khatry, SC LeClerq, RK Adhikari, SR Shrestha and GL Darmstadt. Verbal autopsy methods to ascertain birth asphyxia deaths in a community-based setting in southern Nepal. Pediatrics 2008; 121, e1372-e1380.

[9] N Soleman, D Chandramohan and K Shibuya. Verbal autopsy: Current practices and challenges. Bull. World Health Organ. 2006; 84, 239-45.

[10] WHO. Verbal Autopsy Standard: Ascertaining and Attributing cause of Death. World Health Organization, France, 2007.

[11] J Pattaraarchachai, C Rao, W Polprasert, Y Porapakkham, W Pao-In, N Singwerathum and AD Lopez. Cause-specific mortality patterns among hospital deaths in Thailand: Validating routine death certification. Popul. Health Metrics 2010; 8, 12.

[12] W Polprasert, C Rao, T Adair, J Pattaraarchachai, Y Porapakkham and AD Lopez. Cause-of-death ascertainment for deaths that occur outside hospitals in Thailand: Application of verbal autopsy methods. Popul. Health Metrics 2010; 8, 13.

[13] A Chutinantakul, P Tongkumchum, K Bundhamcharoen and V Chongsuvivatwong. Correcting and estimating HIV mortality in Thailand based on 2005 verbal autopsy data focusing on demographic factors, 1996-2009. Popul. Health Metrics 2014; 12, 25.

[14] N Klinjun, A Lim and K Bundhamcharoen. A logistic regression model for estimating transport accident deaths using verbal autopsy data. Asia Pac. J. Public Health 2015; 27, 286-92.

[15] S Waeto, N Pipatjaturon, P Tongkumchum, C Choonpradub, R Saelim and N Makaje. Estimating liver cancer deaths in Thailand based on verbal autopsy study. J. Res. Health Sci. 2013; 14, 18-22. 